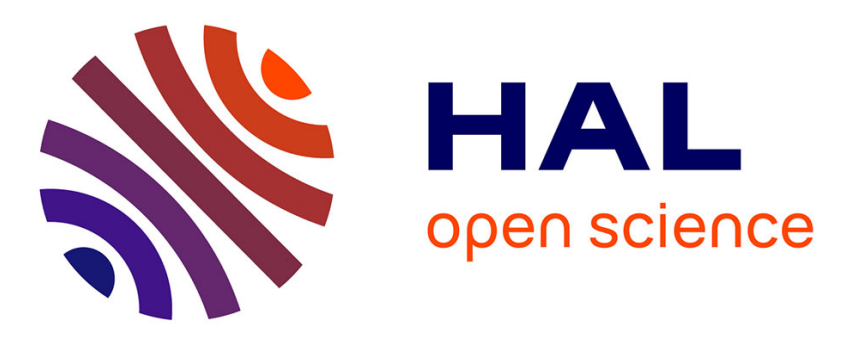

\title{
Waveguide solutions for a nonlinear Schrödinger equation with mixed dispersion
}

Denis Bonheure, Robson Nascimento

\section{To cite this version:}

Denis Bonheure, Robson Nascimento. Waveguide solutions for a nonlinear Schrödinger equation with mixed dispersion. Contributions to Nonlinear Elliptic Equations and Systems, 86, Springer, 2015, Progress in Nonlinear Differential Equations and Their Applications, 10.1007/978-3-319-19902-3_4 . hal-01182833

\section{HAL Id: hal-01182833 \\ https://hal.science/hal-01182833}

Submitted on 3 Aug 2015

HAL is a multi-disciplinary open access archive for the deposit and dissemination of scientific research documents, whether they are published or not. The documents may come from teaching and research institutions in France or abroad, or from public or private research centers.
L'archive ouverte pluridisciplinaire HAL, est destinée au dépôt et à la diffusion de documents scientifiques de niveau recherche, publiés ou non, émanant des établissements d'enseignement et de recherche français ou étrangers, des laboratoires publics ou privés.

$$
\text { Copyright }
$$




\title{
WAVEGUIDE SOLUTIONS FOR A NONLINEAR SCHRÖDINGER EQUATION WITH MIXED DISPERSION
}

\author{
DENIS BONHEURE AND ROBSON NASCIMENTO
}

Ao nosso amigo Djairo com admiraçao

\begin{abstract}
In this note we provide some simple results for the 4NLS model

$$
i \partial_{t} \psi+\Delta \psi+|\psi|^{2 \sigma} \psi-\gamma \Delta^{2} \psi=0,
$$

where $\gamma>0$. Our aim is to partially complete the discussion on waveguide solutions in [11, Section 4.1]. In particular, we show that in the model case with a Kerr nonlinearity $(\sigma=1)$, the least energy waveguide solution $\psi(t, x)=$ $\exp (i \alpha t) u(x)$ with $\alpha>0$ is unique for small $\gamma$ and qualitatively behaves like the waveguide solution of NLS. On the contrary, oscillations arise at infinity when $\gamma$ is too large.
\end{abstract}

\section{INTRODUCTION}

The standard model for propagation of laser beams is the $2 D$ Schrödinger equation with Kerr nonlinearity

$$
i \partial_{t} \psi+\Delta \psi+|\psi|^{2} \psi=0, \quad \psi(x, y, 0)=\psi_{0}(x, y) .
$$

It is well known that this equation can become singular at finite time, see for instance [11] and the classical references therein. Karpman and Shagalov [16] studied the regularization and stabilization effect of a small fourth-order dispersion, namely they considered the equation

$$
i \partial_{t} \psi+\Delta \psi+|\psi|^{2 \sigma} \psi-\gamma \Delta^{2} \psi=0
$$

for some $\gamma>0$, the equation being now considered in $\left[0, \infty\left[\times \mathbb{R}^{N}, N \geq 1\right.\right.$. One of their results shows, by help of some stability analysis and numerical computations, that when $N \sigma \leq 2$, the waveguide solutions are stable for all $\gamma$ and when $2<$ $N \sigma<4$, they are stable for small values of $\gamma$. This result shows that when adding a small fourth-order dispersion term, a new critical exponent/dimension appears. In particular, the Kerr nonlinearity becomes subcritical in dimension 2 and 3 which is obviously an important feature of this extended model.

In [11], Fibich et al. have motivated the study of (1) by recalling that NLS (the nonlinear Schrödinger equation) arises from NLH (the nonlinear Helmholtz equation) as a paraxial approximation. But since NLS can become singular at a finite time, this suggests that some of the small terms, neglected in the paraxial approximation, plays in fact an important role to prevent the blow up. The natural question addressed by Fibich et al. is therefore whether nonparaxiality prevents the collapse. The small fourth-order dispersion coefficient $\gamma$ is then shown to be part of the nonparaxial correction to NLS.

In [11], Fibich et al. showed the role of the new critical exponent $\sigma=4 / N$ in the global existence in time when applying the arguments of Weinstein [25]. The necessary Strichartz estimates follow from Ben-Artzi et al. [1]. A necessary

2000 Mathematics Subject Classification. Primary: 35J60. Secondary: 35B25,35B40,35J10.

Key words and phrases. Schrödinger equation, mixed dispersion NLS, biharmonic NLS, waveguide solutions, ground state solutions. 
condition for existence of waveguide solutions is given in [11, Lemma 4.1], see also the Derrick-Pohozahev identity in Section 6.

The purpose of this short note is to show that classical tools, available in the literature, allow to state the existence and some qualitative properties of least energy waveguide solutions. In particular, a small fourth-order dispersion coefficient does not affect the symmetry, uniqueness and non degeneracy of the least energy waveguide solution at least for a Kerr nonlinearity in dimension $N \leq 3$.

From now on, we focus on standing wave solutions of (1), referred to as waveguide solutions in nonlinear optics, namely on solutions of (1) of the form

$$
\psi(t, x)=\exp (i \alpha t) u(x) .
$$

This ansatz yields the semilinear elliptic equation

$$
\gamma \Delta^{2} u(x)-\Delta u(x)+\alpha u(x)=|u|^{2 \sigma} u(x), \quad x \in \mathbb{R}^{N} .
$$

By scaling the solutions as $v(x)=u\left(\frac{x}{\sqrt[4]{\gamma}}\right)$, it is equivalent to consider the equation

$$
\Delta^{2} v(x)-\beta \Delta v(x)+\alpha v(x)=|v|^{2 \sigma} v(x), \quad x \in \mathbb{R}^{N} .
$$

where $\beta=\frac{1}{\sqrt{\gamma}}$.

It is standard that least energy solutions can be obtained by considering the minimization problem

$$
m_{\mathbb{R}^{N}}:=\inf _{u \in M_{\mathbb{R}^{N}}} J_{\mathbb{R}^{N}}(u)
$$

where

$$
J_{\mathbb{R}^{N}}(u)=\int_{\mathbb{R}^{N}}\left(|\Delta u|^{2}+\beta|\nabla u|^{2}+\alpha|u|^{2}\right) d x
$$

and

$$
M_{\mathbb{R}^{N}}:=\left\{u \in H^{2}\left(\mathbb{R}^{N}\right): \int_{\mathbb{R}^{N}}|u|^{2 \sigma+2} d x=1\right\} .
$$

Indeed, if $u \in M_{\mathbb{R}^{N}}$ achieves the infimum $m=m_{\mathbb{R}^{N}}$, then $u$ weakly solves

$$
\Delta^{2} u-\beta \Delta u+\alpha u=m|u|^{2 \sigma} u .
$$

Henceforth, if $m>0$, then $v=(m)^{\frac{1}{2 \sigma}} u$ solves (3). Moreover $v$ is a least energy solution in the sense that it minimizes the action functional $E: H^{2}\left(\mathbb{R}^{N}\right) \rightarrow \mathbb{R}$ defined by setting

$$
E(u):=\frac{1}{2} J_{\mathbb{R}^{N}}(u)-\frac{1}{2 \sigma+2} \int_{\mathbb{R}^{N}}|u|^{2 \sigma+2} d x
$$

among the set of $\left(H^{2}\right.$ or smoother) solutions or equivalently within the Nehari manifold

$$
\left\{u \in H^{2}\left(\mathbb{R}^{N}\right): E^{\prime}(u)(u)=0\right\} .
$$

We then prove the following results.

Theorem 1. Assume $\alpha>0, \beta>-2 \sqrt{\alpha}$ and $2<2 \sigma+2<\frac{2 N}{N-4}$ if $N \geq 5$. Then problem (3) has a nontrivial least energy solution. If $\beta \geq 2 \sqrt{\alpha}$, then any least energy solution does not change sign, is radially symmetric around some point and strictly radially decreasing.

An existence statement (as well as the information on the sign of the minimizer) is also given in Section 3 and Section 4 when the equation is considered in a bounded domain with Navier boundary conditions. The symmetry properties of the solutions that match the symmetries of the domain are discussed in Section 4. 
When $\beta$ is large, the Laplacian is the driven term in the differential operator in (3) and we therefore expect to recover the uniqueness (up to translations) of the least energy solution. By scaling, we can discuss this issue by looking at least energy solutions of (2) for small $\gamma$. As a preliminary observation, we prove the strong convergence in $H^{1}$ to the unique least energy solution of NLS.

Theorem 2. Assume $2<2 \sigma+2<\frac{2 N}{N-2}$ if $N \geq 3$. If $\gamma_{k} \rightarrow 0$ and $u_{k}$ is a sequence of least energy solutions of $(2)$, then $\left(u_{k}\right)_{k}$ converges (after possible translations) in $H^{1}$ to $u_{0}$, where $u_{0}$ is the unique positive (radially symmetric) solution of the limit problem (2) with $\gamma=0$.

The positive solution of (2) with $\gamma=0$ is unique up to translations. To ensure uniqueness, we have assumed that $u_{0}$ is the positive solution radially decreasing around 0 . For the physical model (2) with $\sigma=1$ in dimension $N \leq 3$, we can improve this convergence to strong convergence in $H^{2}$. The non degeneracy of the least energy waveguide of NLS allows then to invoque the Implicit Function Theorem to prove uniqueness for small $\gamma$.

Theorem 3. Assume $N \leq 3$ and $\sigma=1$. Then there exists $\gamma_{0}>0$ such that if $0<\gamma<\gamma_{0}$, (2) has a unique least energy solution (up to translations). Fixing its maximum at the origin, this solution is radially symmetric and strictly radially decreasing.

An equivalent statement can be proved for the Navier boundary value problem in a ball (and a weaker statement holds for other bounded domains), see Section 6 .

In the $H^{1}$ critical or supercritical regime, the least energy solution should disappear at the limit $\gamma \rightarrow 0$. In fact, if $\frac{2 N}{N-2} \leq 2 \sigma+2<\frac{2 N}{N-4}, N \geq 5$, the least energy solutions are unbounded in $H^{2}$ when $\gamma \rightarrow 0$, see Section 6 .

In contrast with Theorem 1 , when $\beta$ is small in (3), some of the usual properties of the least energy solution of NLS cannot hold. Namely, if one can prove that any least energy solution is radial in that case, then oscillations arise at infinity. These oscillations were suggested in [11]. We focus again on the model equation (2) with $\sigma=1$ in dimension $N \leq 3$. We prove that least energy solutions among radial solutions do oscillate at infinity.

Theorem 4. Suppose that $-2 \sqrt{\alpha}<\beta<2 \sqrt{\alpha}$ and $N \leq 3$. Then every radial least energy solution of (3) with $\sigma=1$ is sign-changing.

This statement shows that when $\beta<2 \sqrt{\alpha}$, least energy solutions cannot be radial and monotone in contrast with the case $\beta \geq 2 \sqrt{\alpha}$. We point out that on a bounded domain, we are not aware of an equivalent statement.

The paper is organized as follows. Section 2 deals with the functional framework and the formulation of the problem on a bounded domain. In Section 3, we prove the existence of a least energy solution in the whole space as well as in bounded domains. In Section 4, we consider the qualitative properties for large $\beta$. Section 5 is dedicated to the proof of Theorem 2 and Theorem 3 while Section 6 contains the proof of Theorem 4. In the last section, we give some concluding remarks.

Notes added in proofs : We thank Jean-Claude Saut for bringing to our attention the reference [5] which deals with an anisotropic mixed dispersion NLS also proposed in [11]. We believe that some arguments from [5] can be used to obtain the exponential decay of the ground state at least in some particular cases.

We also mention the very recent preprint [6] where the first theoretical proof of blow-up is obtained for the biharmonic NLS as well as a new Fourrier rearrangement is proposed in the Appendix. This rearrangement decreases the $L^{2}$-norm of $(-\Delta u)^{s}$ 
for every $s \geq 0$ and is therefore adequate to deal with polyharmonic as well as fractional equations. Applied to our problem, it completes Theorems 1 and 4 in the following way. Assuming $\beta \geq 0$ and $\sigma \in \mathbb{N}_{0}$ (including therefore the physical case $\sigma=1$ ), any ground state solution of (3) is radially symmetric. As a consequence of Theorem 4, assuming $\sigma \in \mathbb{N}_{0}$ and $0 \leq \beta<2 \sqrt{\alpha}$, any ground state is radially oscillatory at infinity. When $\sigma$ is not an integer, the radial symmetry remains an open question in the range $\beta<2 \sqrt{\alpha}$ though the natural conjecture is that radial symmetry holds for every $\sigma$ and every $\beta$ in the range covered by Theorem 1 .

\section{FUnCTIONAL FRAMEWORK}

In this section, we settle the functional setting. The natural space for (2) and (3) is $H^{2}\left(\mathbb{R}^{N}\right)$ or $H^{2}(\Omega) \cap H_{0}^{1}(\Omega)$ when we consider the boundary value problem in a bounded domain $\Omega \subset \mathbb{R}^{N}$ with Navier boundary conditions, namely

$$
\left\{\begin{aligned}
\Delta^{2} u-\beta \Delta u+\alpha u & =|u|^{2 \sigma} u, \quad \text { in } \Omega, \\
u=\Delta u & =0, \quad \text { on } \partial \Omega .
\end{aligned}\right.
$$

We therefore set $H_{\Omega}:=H^{2}(\Omega) \cap H_{0}^{1}(\Omega)$ and $H_{\mathbb{R}^{N}}:=H^{2}\left(\mathbb{R}^{N}\right)$. We introduce the following conditions on $\alpha$ and $\beta$ :

(A1) $\alpha>0$ and $\beta>-2 \sqrt{\alpha}$;

$\left(A 1^{\prime}\right) \alpha>-\beta \lambda_{1}(\Omega)-\lambda_{1}^{2}(\Omega)$ and $-2 \lambda_{1}(\Omega)<\beta$;

where $\lambda_{1}(\Omega)$ stands for the first eigenvalue of $-\Delta$ in $H_{0}^{1}(\Omega)$ when $\Omega$ is a bounded domain. Observe that when $|\Omega|$ is large, $\lambda_{1}(\Omega)$ is small. If $\beta$ is negative, $\left(A 1^{\prime}\right)$ is then more restrictive than $(A 1)$. The following lemma follows from standard computations.

Lemma 1. Assume $\Omega$ is a bounded smooth domain and $(A 1)$ or $\left(A 1^{\prime}\right)$ holds. Then $H_{\Omega}$ is a Hilbert space endowed with the inner product defined through

$$
\langle u, v\rangle=\int_{\Omega}(\Delta u \Delta v+\beta \nabla u \nabla v+\alpha u v) d x \quad \forall u, v \in H_{\Omega} .
$$

Proof. From $H^{2}$ elliptic regularity [14, 18], we know that if $u \in H^{2}(\Omega) \cap H_{0}^{1}(\Omega)$, then

$$
\|u\|_{H^{2}} \leq C\|\Delta u\|_{L^{2}}
$$

for some $C>0$ depending on $\Omega$, so that $H_{\Omega}$ is a Hilbert space endowed with the inner product

$$
\langle u, v\rangle_{H_{\Omega}}=\int_{\Omega} \Delta u \Delta v d x \quad \forall u, v \in H_{\Omega} .
$$

It will be enough to show that there exists a constant $C>0$ such that

$$
\int_{\Omega}\left(|\Delta u|^{2}+\beta|\nabla u|^{2}+\alpha|u|^{2}\right) d x \geq C\|u\|_{H_{\Omega}} \quad \forall u \in H_{\Omega} .
$$

Obviously the inequality (7) holds true if we have $\alpha \geq 0$ and $\beta \geq 0$. For $u \in H_{\Omega}$, we can apply Young's inequality to obtain

$$
\begin{aligned}
\|u\|^{2} & =\int_{\Omega}\left(|\Delta u|^{2}+\beta|\nabla u|^{2}+\alpha|u|^{2}\right) d x \\
& =\int_{\Omega}\left(|\Delta u|^{2}-\beta u \Delta u+\alpha|u|^{2}\right) d x \\
& \geq\left(1+\frac{\beta}{2 \epsilon}\right) \int_{\Omega}|\Delta u|^{2} d x+\left(\alpha+\frac{\beta \epsilon}{2}\right) \int_{\Omega}|u|^{2} d x
\end{aligned}
$$


for every $\epsilon>0$. We have to distinguish two cases. If we can choose $\epsilon>0$ such that both terms in the right hand side of (8) are positive, then we are done. This ends the proof if $\beta>-2 \sqrt{\alpha}$, namely if ( $A 1)$ holds. If

we write

$$
1+\frac{\beta}{2 \epsilon}>0 \quad \text { and } \quad \alpha+\frac{\beta \epsilon}{2}<0,
$$

$$
\|u\|^{2} \geq\left(1+\frac{\beta}{2 \epsilon}\right)\left[\int_{\Omega}|\Delta u|^{2} d x+g(\epsilon) \int_{\Omega}|u|^{2} d x\right]
$$

where

$$
g(\epsilon)=\frac{\alpha+\beta \epsilon / 2}{1+\beta / 2 \epsilon}
$$

Recalling Poincaré inequality

$$
\int_{\Omega}|\Delta u|^{2} d x \geq \lambda_{1}^{2}(\Omega) \int_{\Omega} u^{2} d x \quad \forall u \in H_{\Omega}
$$

we can complete the proof if

$$
g(\epsilon)>-\lambda_{1}^{2}(\Omega)
$$

for some $\epsilon>0$. When $\beta>-2 \lambda_{1}(\Omega)$, this condition can be fulfilled if

$$
\alpha>-\beta \lambda_{1}(\Omega)-\lambda_{1}^{2}(\Omega)
$$

while if $\beta \leq-2 \lambda_{1}(\Omega)$, we recover the condition

$$
-2 \sqrt{\alpha}<\beta .
$$

In the case $\Omega=\mathbb{R}^{N}$, the same arguments show that $(A 1)$ implies

$$
\langle u, v\rangle=\int_{\Omega}(\Delta u \Delta v+\beta \nabla u \nabla v+\alpha u v) d x
$$

is a scalar product on $H_{\mathbb{R}^{N}}$. Elliptic regularity can be used here to ensure that

$$
\left(1+\frac{\beta}{2 \epsilon}\right) \int_{\mathbb{R}^{N}}|\Delta u|^{2} d x+\left(\alpha+\frac{\beta \epsilon}{2}\right) \int_{\mathbb{R}^{N}}|u|^{2} d x
$$

is a norm on $H^{2}\left(\mathbb{R}^{N}\right)$ as soon as $1+\frac{\beta}{2 \epsilon}>0$ and $\alpha+\frac{\beta \epsilon}{2}>0$. This yields the following lemma.

Lemma 2. Assume that (A1) holds. Then the bilinear form

$$
\langle u, v\rangle=\int_{\Omega}(\Delta u \Delta v+\beta \nabla u \nabla v+\alpha u v) d x \quad \forall u, v \in H_{\mathbb{R}^{N}},
$$

is an inner product on $H_{\mathbb{R}^{N}}$.

\section{EXISTENCE OF MINIMIZERS}

In this section, we handle the minimization problem (4). We start with the simpler case of a bounded domain. In this case, the minimization problem writes

$$
m_{\Omega}:=\inf _{u \in M_{\Omega}} J_{\Omega}(u)
$$

where

and

$$
J_{\Omega}(u)=\int_{\Omega}\left(|\Delta u|^{2}+\beta|\nabla u|^{2}+\alpha|u|^{2}\right) d x
$$

$$
M_{\Omega}:=\left\{u \in H_{\Omega}: \int_{\Omega}|u|^{2 \sigma+2} d x=1\right\} .
$$


In the case of a bounded domain, it is standard to prove that $m_{\Omega}$ is achieved when $2 \sigma+2$ is a subcritical exponent because $J_{\Omega}$ is the square of a norm on $H_{\Omega}$ and we can rely on the compactness of the embedding of $H_{\Omega}$ into $L^{2 \sigma+2}(\Omega)$. Moreover, since $m_{\Omega}$ is clearly positive, we deduce that $v=\left(m_{\Omega}\right)^{\frac{1}{2 \sigma}} u$ solves $\left(P_{\beta}\right)$. Moreover $v$ is a least energy solution in the sense that it minimizes the action functional $E_{\Omega}: H_{\Omega} \rightarrow \mathbb{R}$ defined by

$$
E_{\Omega}(u):=\frac{1}{2} J_{\Omega}(u)-\frac{1}{2 \sigma+2} \int_{\Omega}|u|^{2 \sigma+2} d x
$$

among the set of $\left(H^{2}\right.$ or smoother) solutions or equivalently within the Nehari manifold

$$
\left\{u \in H_{\Omega}: E_{\Omega}^{\prime}(u)(u)=0\right\} .
$$

Theorem 5. Assume $\Omega$ is a bounded smooth domain and $(A 1)$ or $\left(A 1^{\prime}\right)$ holds. Suppose moreover that $2<2 \sigma+2<\frac{2 N}{N-4}$ if $N \geq 5$. Then problem $\left(P_{\beta}\right)$ has a nontrivial least energy solution.

To handle the case of $\Omega=\mathbb{R}^{N}$, since we cannot use sign information, nor symmetry, we follow the celebrated method of concentration-compactness of P.L. Lions. We give a sketchy proof since classical arguments apply. All the details can easily be reconstructed from Kavian [17, Chapitre 8 - Exemple 8.5] with minor and obvious modifications with respect to the case treated therein.

Proof of the existence part in Theorem 1. We introduce

$$
M_{\lambda}=\left\{u \in H^{2}\left(\mathbb{R}^{N}\right): \int_{\mathbb{R}^{N}}|u|^{2 \sigma+2} d x=\lambda\right\}
$$

where $\lambda>0$ is fixed and we consider the minimization problem

$$
m_{\lambda}:=\inf _{u \in M_{\lambda}} J_{\mathbb{R}^{N}}(u)
$$

where $J_{\mathbb{R}^{N}}(u)$ is defined as in $(5)$.

Let $\left(u_{k}\right)_{k} \subset M_{\lambda}$ be such that $J_{\mathbb{R}^{N}}\left(u_{k}\right) \rightarrow m_{\lambda}$. Then, $\left(u_{k}\right)_{k}$ is bounded in $H^{2}\left(\mathbb{R}^{N}\right)$ and $\int_{\mathbb{R}^{N}}\left|u_{k}\right|^{2 \sigma+2}=\lambda$. Thus, we can apply P.L. Lions' concentrationcompactness lemma to the sequence $\left(\rho_{k}\right)_{k}=\left(\int_{\mathbb{R}^{N}}\left|u_{k}\right|^{2 \sigma+2}\right)_{k}$, see [21, Lemma I. 1]. Since $m_{\lambda}=\lambda^{\frac{1}{\sigma+1}} m_{1}$, we have $m_{\lambda}>0$ for all $\lambda>0$ and therefore, for all $R>0$, the sequence

$$
Q_{k}(R):=\sup _{y \in \mathbb{R}^{N}} \int_{B_{R}(y)}\left|u_{k}(x)\right|^{2 \sigma+2} d x
$$

does not converge to zero. Namely, vanishing is ruled out.

Since $2 \sigma+2>2$, we have, for $0<\theta<\lambda$,

$$
\lambda^{\frac{1}{\sigma+1}}<\theta^{\frac{1}{\sigma+1}}+(\lambda-\theta)^{\frac{1}{\sigma+1}}
$$

which yields

$$
\left.m_{\lambda}<m_{\theta}+m_{\lambda-\theta}, \quad \forall \theta \in\right] 0, \lambda[.
$$

Then dichotomy is ruled out using classical truncation arguments.

Therefore, the compactness holds for $\rho_{k}$, i.e., going to a subsequence of $\left(u_{k}\right)$ if necessary, there exists a sequence $\left(y^{k}\right) \subset \mathbb{R}^{N}$ such that for every $\varepsilon>0$, there exists $R>0$ such that

$$
\int_{B_{R}\left(y^{k}\right)}\left|u_{k}\right|^{2 \sigma+2} d x>\lambda-\varepsilon
$$

Setting $w_{k}(x):=u_{k}\left(x+y^{k}\right)$, we have that $\left(w_{k}\right)$ is also a minimizing sequence for $m_{\lambda}$. Then, up to a subsequence, $w_{k}$ weakly converges in $H^{2}\left(\mathbb{R}^{N}\right)$ to $w \in M_{\lambda}$ and $J_{\mathbb{R}^{N}}(w)=m_{\lambda}$. This concludes the proof of the existence in Theorem 1 . 
Remark 1. When $\beta \geq 2 \sqrt{\alpha}$, we can avoid the use of the concentration-compactness lemma. Indeed, take a minimizing sequence $\left(u_{k}\right)_{k} \subset H^{2}\left(\mathbb{R}^{N}\right)$ for $m$. Then, let us set $f_{k}:=-\Delta u_{k}+\beta u_{k} / 2$ and define $v_{k} \in H^{2}\left(\mathbb{R}^{N}\right)$ to be the strong solution of $-\Delta v_{k}+\beta v_{k} / 2=\left|f_{k}\right|^{*}$ in $\mathbb{R}^{N}$, where $\left|f_{k}\right|^{*}$ denotes the Schwarz symmetrization of $\left|f_{k}\right|$. Thus for each $k \in \mathbb{N}$, we have $v_{k} \in H_{\text {rad }}^{2}\left(\mathbb{R}^{N}\right)$ which is the space of $H^{2}$ functions that are radially symmetric around the origin. Then a particular case of [3, Lemma 3.4] implies

$$
\begin{aligned}
J\left(\frac{v_{k}}{\left|v_{k}\right|_{2 \sigma+2}}\right) & =\frac{\int_{\mathbb{R}^{N}}\left(-\Delta v_{k}+\beta v_{k} / 2\right)^{2} d x-\left(\beta^{2} / 4-\alpha\right) \int_{\mathbb{R}^{N}} v_{k}^{2} d x}{\left|v_{k}\right|_{2 \sigma+2}^{2}} \\
& \leq \frac{\int_{\mathbb{R}^{N}}\left(-\Delta u_{k}+\beta u_{k} / 2\right)^{2} d x-\left(\beta^{2} / 4-\alpha\right) \int_{\mathbb{R}^{N}} u_{k}^{2} d x}{\left|u_{k}\right|_{2 \sigma+2}^{2}} .
\end{aligned}
$$

Using the compact embedding of $H_{r a d}^{2}\left(\mathbb{R}^{N}\right)$ into $L^{2 \sigma+2}\left(\mathbb{R}^{N}\right)$, see for instance $[20$, Théorème II.1], it follows that $\left(v_{k}\right)_{k}$ weakly converges in $H^{2}$ to some $v \in M$ and the remaining arguments are standard.

\section{Sign AND SYMmetry}

In order to investigate the symmetry properties of a fourth order equation with Navier boundary conditions or in the whole space, it is natural to ask if the equation may be rewritten as a cooperative system. If this is the case, then the moving plane procedure applies, see the work of Troy [23] in the case of a bounded domain or de Figueiredo-Yang [13] (if we assume exponential decay) and Busca-Sirakov [7] (without assuming exponential decay) when $\Omega=\mathbb{R}^{N}$. Observe that when $\alpha>0$ and $|\beta| \geq 2 \sqrt{\alpha}$, we can indeed write the equation as a cooperative system

$$
-\Delta u+\frac{\beta}{2} u-v=0, \quad-\Delta v+\left(\alpha-\frac{\beta^{2}}{4}\right) u+\frac{\beta}{2} v=|u|^{2 \sigma} u .
$$

To prove that least energy solutions do not change sign, we use the minimality combined to the classical maximum principle for a single equation. The argument goes back to van der Vorst, see for instance [24]. We sketch it for completeness to emphasize the role of the assumption $|\beta| \geq 2 \sqrt{\alpha}$.

Lemma 3. Assume that $|\beta| \geq 2 \sqrt{\alpha}$ and $-\lambda_{1}(\Omega)<\beta / 2$ if $\Omega$ is bounded or $\beta>0$ if $\Omega=\mathbb{R}^{N}$. If $u \in H_{\Omega}$ is a minimizer of (4), then

$$
u>0 \quad \text { and } \quad-\Delta u+\beta u / 2>0 \text { in } \Omega,
$$

or else

$$
u<0 \quad \text { and } \quad-\Delta u+\beta u / 2<0 \quad \text { in } \Omega .
$$

Proof. Let $w \in H_{\Omega}$ be such that

$$
\left\{\begin{aligned}
-\Delta w+\beta w / 2 & =|-\Delta u+\beta u / 2|, \quad \text { in } \Omega \\
w & =0, \quad \text { on } \partial \Omega
\end{aligned}\right.
$$

Then

$$
-\Delta(w \pm u)+\beta(w \pm u) / 2 \geq 0 .
$$

Using the strong maximum principle we know that $u$ has a fixed sign if $-\Delta u+\beta / 2 u$ does not change sign. We then argue by contradiction, suppose that $-\Delta u+\beta u / 2$ changes sign. Then $|-\Delta u+\beta u / 2| \neq 0$ and the strong maximum principle implies 
that $w>|u|$. For convenience denote by $|\cdot|_{2 \sigma+2}$ the $L^{2 \sigma+2}$ norm in $\Omega$. Therefore

$$
\begin{aligned}
J_{\Omega}\left(\frac{w}{|w|_{2 \sigma+2}}\right) & =\frac{\int_{\Omega}(-\Delta w+\beta w / 2)^{2} d x-\left(\beta^{2} / 4-\alpha\right) \int_{\Omega} w^{2} d x}{|w|_{2 \sigma+2}^{2}} \\
& <\frac{\int_{\Omega}(-\Delta u+\beta u / 2)^{2} d x-\left(\beta^{2} / 4-\alpha\right) \int_{\Omega} u^{2} d x}{|u|_{2 \sigma+2}^{2}}
\end{aligned}
$$

which contradicts the minimality of $u$. Observe that the last inequality holds because the numerator is nonnegative.

Remark 2. In the case of a bounded domain $\Omega$ and $0<\alpha \leq \lambda_{1}(\Omega)^{2}$, we then know the sign of the least energy solutions of $\left(P_{\beta}\right)$ for values of $\beta \in\left(-2 \lambda_{1}(\Omega),-2 \sqrt{\alpha} \cup\right.$ $[2 \sqrt{\alpha}, \infty)$. For $\Omega$ bounded, we do not know if the least energy solutions change sign for $\beta \in(-2 \sqrt{\alpha}, 2 \sqrt{\alpha})$. Section 6 deals with the case $\Omega=\mathbb{R}^{N}$ under the assumption that the minimizer is radial.

Proof of Theorem 1 continued. Existence was proved in Section 3 while we just proved in Lemma 3 that any least energy solution does not change sign.

Writing $f(u, v)=\left(\frac{\beta^{2}}{4}-\alpha\right) u-\frac{\beta}{2} v+|u|^{2 \sigma} u$ and $g(u, v)=v-\frac{\beta}{2} u$, the equation is equivalent to the cooperative system

$$
\Delta u+g(u, v)=0, \quad \Delta v+f(u, v)=0 .
$$

We are in the setting of Busca-Sirakov [7] and [7, Theorem 2] applies. Observe that clearly $u$ and $v$ must be symmetric with respect to the same point.

In the case of a bounded domain, we have proved so far the following result for $\left(P_{\beta}\right)$.

Theorem 6. Assume $\Omega$ is a bounded smooth domain and $(A 1)$ or $\left(A 1^{\prime}\right)$ holds. Suppose moreover that $2<2 \sigma+2<\frac{2 N}{N-4}$ if $N \geq 5$. Then problem $\left(P_{\beta}\right)$ has a nontrivial least energy solution. If in addition $|\beta| \geq 2 \sqrt{\alpha}$ and $-\lambda_{1}(\Omega)<\beta / 2$, then any least energy solution does not change sign. If $\Omega$ is a ball, then any least energy solution is radially symmetric and strictly radially decreasing.

Proof. Existence has been achieved in Theorem 5 while the sign information follows from Lemma 3. If $\Omega$ is a ball, the symmetry of the minimizer follows from $[23$, Theorem 1].

We point out that the condition $|\beta| \geq 2 \sqrt{\alpha}$ is crucial to rewrite the problem $\left(P_{\beta}\right)$ as a cooperative system. In fact, we can deal more generally with smooth bounded or unbounded domain $\Omega$ with some symmetries. Then the symmetry properties of the solutions of constant sign can be deduced from the moving plane method adapted to cooperative systems in [23].

\section{THE EFFECT OF A SMALL FOURTH ORDER DISSIPATION}

In this section, we study the behaviour of minimizers of (4) when the coefficient of fourth order dissipation tends to zero. We assume throughout the section that $\alpha>0$ and we choose the norm on $H^{1}\left(\mathbb{R}^{N}\right)$ defined through

$$
\|u\|_{H^{1}}^{2}=\int_{\Omega}\left(|\nabla u|^{2}+\alpha|u|^{2}\right) d x .
$$

We recall that the problem

$$
\Delta^{2} v(x)-\beta \Delta v(x)+\alpha v(x)=|v|^{2 \sigma} v(x), \quad x \in \mathbb{R}^{N}
$$


is equivalent to

$$
\gamma \Delta^{2} u(x)-\Delta u(x)+\alpha u(x)=|u|^{2 \sigma} u(x), \quad x \in \mathbb{R}^{N} .
$$

by scaling the solutions as $u(x)=v\left(\frac{x}{\sqrt{\beta}}\right)$ where $\gamma=1 / \beta^{2}$. As before we consider the associated minimization problem

$$
m_{\gamma}=\inf _{u \in M} J_{\gamma}(u)
$$

where

$$
M=\left\{u \in H_{\Omega}: \int_{\Omega}|u|^{2 \sigma+2} d x=1\right\}
$$

and

$$
J_{\gamma}(u)=\int_{\Omega}\left(\gamma|\Delta u|^{2}+|\nabla u|^{2}+\alpha|u|^{2}\right) d x .
$$

When $\Omega=B_{R}$ or $\Omega=\mathbb{R}^{N}$, the results of the previous sections imply that when $\gamma \leq \frac{1}{4 \alpha}$, any minimizer is radially symmetric and strictly radially decreasing (after a possible translation in the case $\Omega=\mathbb{R}^{N}$ ). In the case $\Omega=\mathbb{R}^{N}$, we assume from now on that the maximum of any minimizer has been translated to the origin.

For $\gamma=0$, the associated minimization problem is

$$
m_{0}=\inf _{u \in M_{0}} J_{0}(u)
$$

where

and

$$
M_{0}=\left\{u \in H_{0}^{1}(\Omega): \int_{\Omega}|u|^{2 \sigma+2} d x=1\right\}
$$

$$
J_{0}(u)=\int_{\Omega}\left(|\nabla u|^{2}+\alpha|u|^{2}\right) d x .
$$

Assume $2<2 \sigma+2<\frac{2 N}{N-2}$ if $N \geq 3, \Omega=B_{R}$ or $\Omega=\mathbb{R}^{N}$ and let $u_{0}$ be the unique minimizer of $J_{0}$ in $M_{0}$. We refer to $[9,19,15]$ for the uniqueness property (in the case $\Omega=\mathbb{R}^{N}$, we fix the maximum of the solution at the origin to achieve uniqueness). We first prove that if $\gamma_{k} \rightarrow 0$, then any sequence $\left(u_{k}\right)_{k}$ of minimizer of $J_{\gamma_{k}}$ converge strongly in $H^{1}$ to $u_{0}$. A similar statement obviously holds for other bounded domains except that uniqueness of the minimizer does not hold in general so that in the conclusion, we can only state that we have convergence to one minimizer, see Theorem 7 .

Proposition 1. Assume $2<2 \sigma+2<\frac{2 N}{N-2}$ if $N \geq 3, \Omega=B_{R}$ or $\Omega=\mathbb{R}^{N}$. There exists $C>0$ such that for every $\gamma>0$, we have

$$
m_{0} \leq m_{\gamma} \leq m_{0}+C \gamma
$$

Moreover, if $\gamma_{k} \rightarrow 0$ and $\left(u_{k}\right)_{k}$ is a sequence such that $J_{\gamma_{k}}\left(u_{k}\right)=m_{\gamma_{k}}$, then $u_{k} \rightarrow u_{0}$ strongly in $H^{1}$.

Proof. The estimate of $m_{\gamma}$ is clear since by elliptic regularity, we easily infer that $u_{0} \in H^{2}(\Omega)$. Therefore, we have

$$
m_{\gamma} \leq J_{\gamma}\left(u_{0}\right)=\gamma \int_{\Omega}\left|\Delta u_{0}\right|^{2} d x+J_{0}\left(u_{0}\right) \leq C \gamma+m_{0}
$$

whereas taking any minimizer $u_{\gamma}$ for $m_{\gamma}$, we get

$$
m_{\gamma}=J_{\gamma}\left(u_{\gamma}\right)=\gamma \int_{\Omega}\left|\Delta u_{\gamma}\right|^{2} d x+J_{0}\left(u_{\gamma}\right) \geq m_{0} .
$$

Let $\gamma_{k} \rightarrow 0$ and $\left(u_{k}\right)_{k}$ be a sequence of minimizers for $m_{k}:=m_{\gamma_{k}}$. Then

$$
\int_{\Omega}\left(\left|\nabla u_{k}\right|^{2}+\alpha\left|u_{k}\right|^{2}\right) d x \leq m_{k} \leq m_{0}+C \gamma_{k} \rightarrow m_{0}
$$


Since we know that $u_{k}$ is a radial function, it follows that $u_{k}$ is bounded in $H_{\text {rad }}^{1}(\Omega)$ - the space of $H^{1}$ functions that are radially symmetric around the origin - so that up to a subsequence, $u_{k}$ converges weakly in $H^{1}$ to some $u \in M$. The strong convergence in $L^{2 \sigma+2}$ when $\Omega=\mathbb{R}^{N}$ follows from the compact embedding of $H_{\text {rad }}^{1}\left(\mathbb{R}^{N}\right)$ into $L^{2 \sigma+2}\left(\mathbb{R}^{N}\right)$, see $[22,20]$.

Now, by weak lower semi-continuity, we have

$$
\begin{array}{r}
m_{0} \leq \int_{\Omega}\left(|\nabla u|^{2}+\alpha|u|^{2}\right) d x \leq \liminf _{k \rightarrow \infty} \int_{\Omega}\left(\left|\nabla u_{k}\right|^{2}+\alpha\left|u_{k}\right|^{2}\right) d x \\
\leq \limsup _{k \rightarrow \infty} \int_{\Omega}\left(\left|\nabla u_{k}\right|^{2}+\alpha\left|u_{k}\right|^{2}\right) d x=m_{0} .
\end{array}
$$

Hence the convergence is strong in $H^{1}$ and $u$ is a minimizer for $m_{0}$. By uniqueness, $u=u_{0}$ and the whole sequence converge.

In the model case with a Kerr nonlinearity in dimension $N \leq 3$, we can improve this convergence.

Proposition 2. Assume $\Omega=\mathbb{R}^{N}, \sigma=1$ and $N \leq 3$. If $\gamma_{k} \rightarrow 0$ and $\left(u_{k}\right)_{k}$ is a sequence such that $J_{\gamma_{k}}\left(u_{k}\right)=m_{\gamma_{k}}$, then $u_{k} \rightarrow u_{0}$ strongly in $H^{2}$.

Proof. To fix the ideas, we deal with the case $N=3, N=2$ being similar. The starting point is an a priori bound in $H^{1}$ and the strategy is to end up with an a priori $H^{4}$-bound. We already know from Proposition 1 that $u_{k}$ converges to $u_{0}$ strongly in $H^{1}$. To improve the convergence, we use the Euler-Lagrange equation

$$
\gamma_{k} \Delta^{2} u_{k}-\Delta u_{k}+\alpha u_{k}=m_{k} u_{k}^{3}
$$

where $m_{k}=m_{\gamma_{k}}$. We can assume $\gamma_{k} \leq 1$ and $m_{k} \in\left[m_{0}, m_{0}+C\right]$.

Bound in $H^{1}$. Since $u_{k}$ is a minimizer, we can assume

$$
\left\|u_{k}\right\|_{H^{1}} \leq m_{0}+C .
$$

This also provides an a priori bound in $L^{q}$ for every $q \in[2,6]$.

Bound in $H^{2}$. We denote $v_{k}=-\gamma_{k} \Delta u_{k}$. Then $v_{k}$ solves

$$
-\Delta v_{k}+\frac{1}{\gamma_{k}} v_{k}=w_{k}
$$

where $w_{k}:=m_{k} u_{k}^{3}-\alpha u_{k}$. Since $J_{\gamma_{k}}\left(u_{k}\right) \leq m_{0}+C$, we infer that $v_{k} \rightarrow 0$ strongly in $L^{2}$. In particular, $\left(v_{k}\right)_{k}$ is bounded in $L^{2}$. Observe also that $\left(w_{k}\right)_{k}$ is a priori bounded in $L^{2}$. Now, by elliptic regularity, we infer that $v_{k} \in H^{2}\left(\mathbb{R}^{3}\right)$ with a bound that does not depend on $k$. Indeed, since $\frac{1}{\gamma_{k}} \geq 1$, we get this a priori bound as in Krylov [18, Chapter 1, Theorems $6.4 \& 6.5]$. Now, from this a priori $H^{2}$-bound on $\left(v_{k}\right)_{k}$ and the Euler equation

$$
-\Delta u_{k}+\alpha u_{k}=m_{k} u_{k}^{3}+\Delta v_{k}
$$

we deduce that $\left(u_{k}\right)_{k}$ is a priori bounded in $H^{2}\left(\mathbb{R}^{3}\right)$ as well.

Bound in $H^{4}$. It is straightforward to check that the $H^{2}$-bound on $u_{k}$ implies that $w_{k} \in L^{2}\left(\mathbb{R}^{3}\right)$ and $\Delta w_{k} \in L^{2}\left(\mathbb{R}^{3}\right)$. Then, elliptic regularity implies $w_{k}$ is bounded in $H^{2}$ as well. Using again (10), we now infer that $v_{k} \in H^{4}$ with a bound independent of $k$, arguing as in Krylov for $H^{m+2}$ regularity [18, Chapter 1, Theorem $7.5 \&$ Corollary 7.6]. Looking at (11) again, we have that the right hand side is bounded in $H^{2}$, whence $u_{k} \in H^{4}$ with a bound independent of $k$. 
Conclusion. Observe now that we can use the equation (11) to conclude. Since $-\Delta v_{k}=\gamma_{k} \Delta^{2} u_{k} \rightarrow 0$ strongly in $L^{2}$, we conclude that

$$
m_{k} u_{k}^{3}+\Delta v_{k} \rightarrow m_{0} u_{0}^{3}
$$

strongly in $L^{2}$ and elliptic regularity applied to (11) implies that the convergence of $u_{k}$ to $u_{0}$ is actually strong in $H^{2}$.

Now that we have proved the strong convergence in $H^{2}$ to the unique minimizer for $\gamma=0$, we can use its non degeneracy to apply the Implicit Function Theorem. This yields Theorem 3 .

Proof of Theorem 3. We start by setting $X:=H_{\text {rad }}^{2}\left(\mathbb{R}^{3}\right)$ and $Y:=H^{-2}\left(\mathbb{R}^{3}\right)$. Let $F: \mathbb{R}^{+} \times X \rightarrow Y$ be the operator defined (in the sense of distributions) by

$$
F(\gamma, u)=\gamma \Delta^{2} u-\Delta u+\alpha u-|u|^{2} u .
$$

Namely, for every $v \in H^{2}\left(\mathbb{R}^{3}\right)$, we have

$$
F(\gamma, u)(v)=\int_{\mathbb{R}^{3}}\left(\gamma \Delta u \Delta v+\nabla u \nabla v+\alpha u v-|u|^{2} u v\right) d x .
$$

Obviously $F\left(0, \sqrt{m_{0}} u_{0}\right)=0$. Also, $F$ is continuously differentiable in a neighbourhood of $\left(0, \sqrt{m_{0}} u_{0}\right)$ with $D_{u} F(\gamma, u) \in \mathcal{L}(X, Y)$ defined by

$$
D_{u} F(\gamma, u) v=\gamma \Delta^{2} v-\Delta v+\alpha v-3|u| u v, \quad \forall v \in X
$$

i.e.

$$
D_{u} F(\gamma, u) v[w]=\int_{\mathbb{R}^{3}}(\gamma \Delta v \Delta w+\nabla v \nabla w+\alpha v w-3|u| u v w) d x, \quad \forall v, w \in X .
$$

We thus have in the distributional sense

$$
L(v):=D_{u} F\left(0, \sqrt{m_{0}} u_{0}\right) v=-\Delta v+\alpha v-3 m_{0} u_{0}^{2} v .
$$

It is well-known that the kernel of $L$ is of dimension 3 when considered in $H^{2}\left(\mathbb{R}^{3}\right)$ and it is spanned by the partial derivatives of $u_{0}$. In particular, the kernel of $L$ restricted to $H_{\text {rad }}^{2}\left(\mathbb{R}^{3}\right)$ is trivial and $L: X \rightarrow Y$ is one-to-one. We refer for instance to $[19,15,8]$. Moreover, it follows from the Open Mapping Theorem that $L^{-1}: Y \rightarrow X$ is continuous.

Since the linear map $L$ is a homeomorphism, we can apply the Implicit Function Theorem. Namely, there exists $\gamma_{0}>0$ and an open set $U_{0} \subset X$ that contains $\sqrt{m_{0}} u_{0}$ such that for every $\gamma \in\left[0, \gamma_{0}[\right.$, the equation $F(\gamma, u)=0$ has a unique solution $u_{\gamma} \in U_{0}$ and the curve

$$
\Gamma:\left[0, \gamma_{0}\left[\rightarrow H^{2}\left(\mathbb{R}^{3}\right): \gamma \mapsto u_{\gamma}\right.\right.
$$

is of class $C^{1}$.

Now suppose that the uniqueness of least energy solutions fails in every interval $(0, \gamma)$. We can then construct two sequences in $M$ of least energy solutions along a sequence $\gamma_{k}$ converging to 0 . We call them $\left(u_{k}\right)_{k}$ and $\left(v_{k}\right)_{k}$ whereas $m_{k}$ is their common energy. By assumption, $u_{k} \neq v_{k}$. Since $\gamma_{k} \rightarrow 0$, we know that $u_{k}$ and $v_{k}$ are radially symmetric. Since these two sequences converge in $H^{2}$ to $u_{0}$ as $k \rightarrow \infty$, we have

$$
\sqrt{m_{k}} u_{k}, \sqrt{m_{k}} v_{k} \rightarrow \sqrt{m_{0}} u_{0}
$$

where the convergence is strong in $H^{2}$. Then, for $k$ large enough, there exist two solutions of the equation $F\left(\gamma_{k}, u\right)=0$ in $U_{0}$ with $\gamma_{k}<\gamma_{0}$. This is a contradiction and ends the proof. 
We now state the counterpart of Theorem 3 for the boundary value problem in a bounded domain $\Omega \subset \mathbb{R}^{N}$ with Navier boundary conditions, namely

$$
\left\{\begin{aligned}
\gamma \Delta^{2} u-\Delta u+\alpha u & =|u|^{2 \sigma} u, \quad \text { in } \Omega, \\
u=\Delta u & =0, \quad \text { on } \partial \Omega .
\end{aligned}\right.
$$

We assume in the next statement that $\Omega$ is smooth. We have not searched to optimize the required regularity of the boundary. At some point, we need to take two partial derivatives into the equation. We assume enough regularity of the boundary so that the solution belongs at least to $H^{6}(\Omega)$. One could work with interior regularity which requires less regularity on the boundary but since our main motivation is to cover the case of a ball, working with global regularity is fine for our purpose as the ball has the regularity required.

Theorem 7. Assume $\Omega \subset \mathbb{R}^{N}$ is a smooth bounded domain of class $C^{6}$ and $3 \leq$ $2 \sigma+2<\frac{2 N}{N-2}$ if $3 \leq N \leq 5$. If $\gamma_{k} \rightarrow 0$ and $\left(u_{k}\right)_{k}$ is a sequence of least energy solutions of $\left(P_{\gamma_{k}}\right)$, then, up to a subsequence, $u_{k}$ converges strongly in $H^{2}$ to some minimizer $u_{0}$ for $m_{0}$. If, in addition, $\Omega$ is a ball, then there exists $\gamma_{0}>0$ such that if $0<\gamma<\gamma_{0}$, the problem $\left(P_{\gamma}\right)$ has a unique least energy solution. This solution is radially symmetric and strictly radially decreasing.

Proof. Step 1. Global regularity. Using elliptic regularity [14, Theorems 8.12 \& 8.13], we easily infer that the solutions $u_{k}$ are smooth, namely at least $H^{6}(\Omega)$. Indeed, one can write the equation as a double Dirichlet problem

$$
\begin{gathered}
-\Delta u_{k}=\phi_{k}, \quad u_{k}=0 \quad \text { on } \partial \Omega, \\
-\gamma_{k} \Delta \phi_{k}+\phi_{k}=m_{k}\left|u_{k}\right|^{2 \sigma} u_{k}-\alpha u_{k}, \quad \phi_{k}=0 \text { on } \partial \Omega .
\end{gathered}
$$

Here $\gamma_{k}$ stays fixed and we can start with the fact that $u_{k} \in H^{2}(\Omega)$, without caring about the dependence on $k$. Then the term $m_{k}\left|u_{k}\right|^{2 \sigma} u_{k}-\alpha u_{k} \in L^{2}(\Omega)$ as it can be easily checked from the assumption on $\sigma$ and the embedding of $H^{2}(\Omega)$ into $L^{q}(\Omega)$ for every $q \geq 1$ if $N \leq 4$ and $q \in\left[1, \frac{2 N}{N-4}\right]$ if $N=5$. We therefore infer from [14, Theorems 8.12] that $\phi_{k} \in H^{2}(\Omega)$ which in turn implies that $u_{k} \in H^{4}(\Omega)$ by $[14$, Theorems 8.13]. Now computing $\Delta\left(m_{k}\left|u_{k}\right|^{2 \sigma} u_{k}-\alpha u_{k}\right)$, we realize that it is an $L^{2}$ function and therefore $m_{k}\left|u_{k}\right|^{2 \sigma} u_{k}-\alpha u_{k}$ is an $H^{2}$ function. Indeed, the condition on $\sigma$ ensures the required integrability of $\left|u_{k}\right|^{2 \sigma-1}\left|\nabla u_{k}\right|^{2}$ and $\left|u_{k}\right|^{2 \sigma}\left|\Delta u_{k}\right|$. We then conclude that $\phi_{k}$ belongs in fact at least to $H^{4}$ and therefore $u_{k} \in H^{6}(\Omega)$.

Step 2. Strong convergence in $H^{1}$. Arguing as in the proof of Proposition 1, we infer that there exists a minimizer $u_{0} \in M_{0}$ and a subsequence that we still denote $\left(u_{k}\right)_{k}$ such that $u_{k} \rightarrow u_{0}$ strongly in $H^{1}$. If $\Omega$ is a ball, then $u_{0}$ is the unique minimizer and the whole sequence converge.

Step 3. Strong convergence in $H^{2}$. To improve the convergence, we argue as in the proof of Proposition 2. If $2 \sigma+1 \leq \frac{N}{N-2}$, then we can bootstrap using the $H^{m+2}$ regularity theory. Due to the boundary condition, the argument of Krylov [18, Chapter 1] cannot be applied directly to get higher regularity in general, see [18, Chapter 8]. However, in our case, since we deal with Navier condition, we have that $u_{k}=\Delta u_{k}=0$ on the boundary and therefore the equation $\left(P_{\gamma_{k}}\right)$ tells that $\Delta^{2} u_{k}=0$ on the boundary as well. By Step 1, we can take the Laplacian inside the equation in $\left(P_{\gamma_{k}}\right)$ and use the fact that $\Delta u_{k}$ solves a boundary problem with Navier boundary conditions, namely

$$
\begin{aligned}
\gamma_{k} \Delta^{2}\left(\Delta u_{k}\right)-\Delta\left(\Delta u_{k}\right)+\alpha\left(\Delta u_{k}\right) & =m_{k} f\left(u_{k}\right), \quad \text { in } \Omega, \\
\Delta\left(\Delta u_{k}\right)=\Delta u_{k} & =0, \quad \text { on } \partial \Omega,
\end{aligned}
$$

where

$$
f\left(u_{k}\right)=(2 \sigma+1) \operatorname{sign}\left(u_{k}\right)\left(2 \sigma u_{k}^{2 \sigma-1}\left|\nabla u_{k}\right|^{2}+u_{k}^{2 \sigma} \Delta u_{k}\right) .
$$


Then we can use the $H^{2}$ regularity for the Dirichlet problem associated to the systems

$$
v_{k}=-\gamma_{k} \Delta u_{k} \quad-\Delta v_{k}+\frac{1}{\gamma_{k}} v_{k}=w_{k}
$$

and

$$
y_{k}=\Delta v_{k}=-\gamma_{k} \Delta^{2} u_{k} \quad-\Delta y_{k}+\frac{1}{\gamma_{k}} y_{k}=m_{k} f\left(u_{k}\right),
$$

where $w_{k}=m_{k}\left|u_{k}\right|^{2 \sigma} u_{k}-\alpha u_{k}$ and $f\left(u_{k}\right)$ is defined in (12). Applying [18, Chapter 8 , Theorem 8.7 ] to the second equation of the first system (13), we get an $H^{2}$ a priori bound of $v_{k}$. Now turning to the Dirichlet problem

$$
-\Delta u_{k}+\alpha u_{k}=m_{k}\left|u_{k}\right|^{2 \sigma} u_{k}+\Delta v_{k}, \quad u_{k}=0 \text { on } \partial \Omega,
$$

we deduce that $u_{k}$ is a priori bounded in $H^{2}$ which leads to an $L^{2}$ bound for $f\left(u_{k}\right)$. Applying then [18, Chapter 8, Theorem 8.7 ] on the second equation of the system (14) gives an $H^{2}$ a priori bound of $\Delta v_{k}$. Whence $v_{k}$ is a priori bounded in $H^{4}$. This allows to conclude that $u_{k}$ is a priori bounded in $H^{4}$ because the right hand side of $\Delta(15)$, namely

$$
-\Delta\left(\Delta u_{k}\right)+\alpha \Delta u_{k}=m_{k} f\left(u_{k}\right)+\Delta^{2} v_{k},
$$

is a priori bounded in $L^{2}$. The remaining steps are now as in the proof of Proposition 2 .

If $\frac{N}{N-2}+1<2 \sigma+2<\frac{2 N}{N-2}$, we can only start with a bound in $L^{\frac{2 N}{(N-2)(2 \sigma+1)}}$ on the right hand side of

$$
-\Delta v_{k}+\frac{1}{\gamma_{k}} v_{k}=w_{k}
$$

where we still use the notations $v_{k}=-\gamma_{k} \Delta u_{k}$ and $w_{k}=m_{k}\left|u_{k}\right|^{2 \sigma} u_{k}-\alpha u_{k}$. We therefore need to improve this bound first. Arguing as above (still using [18, Chapter 8 , Theorem 8.7$]$ ), we deduce an a priori bound in $W^{2, q}$ with $q=\frac{2 N}{(N-2)(2 \sigma+1)}$. Then Sobolev embeddings give a better integrability of $w_{k}$ and we can bootstrap until we get an $L^{2}$ a priori bound on $w_{k}$. The strong convergence in $H^{2}$ is then achieved as in the proof of Proposition 2 taking into account the above remark concerning the way to obtain the higher order elliptic regularity. Observe that even if $\frac{N}{N-2}+1<2 \sigma+2$, no additional bootstrap is necessary to derive the $H^{4}$ bound on $u_{k}$ since once we get an a priori $H^{2}$ bound on $u_{k}$, the assumption on $\sigma$ implies that $f\left(u_{k}\right)$ is a priori bounded in $L^{2}$.

Uniqueness in the case $\Omega=B_{R}$. When $\Omega$ is a ball, the arguments used in the proof of Theorem 3 are available. The non degeneracy of $u_{0}$ allows to apply the Implicit Function Theorem to conclude the local uniqueness (in an $H^{2}$ neighbourhood of $u_{0}$ ) for $\gamma$ small. The remaining arguments are then as in the proof of Theorem 3 .

We end up the analysis of the asymptotics for $\gamma \rightarrow 0$ by showing that the least energy solution blows up in $H^{2}$ when $2 \sigma+2$ is $H^{1}$ critical or supercritical. We focus on the case of $\Omega=\mathbb{R}^{N}$.

We first derive the Derrick-Pohozahev identity for minimizers. If $u$ achieves $m_{\gamma}$ in $M$, then, defining $v_{\lambda}$ by $v_{\lambda}(x)=\lambda^{\frac{N}{2 \sigma+2}} u(\lambda x)$, we infer that $f(\lambda):=J_{\gamma}\left(v_{\lambda}\right)$ achieves a local minimum at $\lambda=1$. This yields a Derrick-Pohozahev identity

$$
\begin{aligned}
\gamma(2 N-(2 \sigma+2)(N-4)) \int_{\mathbb{R}^{N}}|\Delta u|^{2} d x+ & (2 N-(2 \sigma+2)(N-2)) \int_{\mathbb{R}^{N}}|\nabla u|^{2} d x \\
& +\alpha(2 N-(2 \sigma+2) N) \int_{\mathbb{R}^{N}}|u|^{2} d x=0 .
\end{aligned}
$$


If $2 \sigma+2 \geq \frac{2 N}{N-4}$, then $u$ must be zero which is obviously a contradiction. This shows that $m_{\gamma}$ is not achieved for $2 \sigma+2 \geq \frac{2 N}{N-4}$.

For $\frac{2 N}{N-2} \leq 2 \sigma+2<\frac{2 N}{N-4}$, the first coefficient in the Derrick-Pohozahev identity is positive whereas the other two are non positive. We can then write

$$
\gamma(2 N-(2 \sigma+2)(N-4)) \int_{\mathbb{R}^{N}}|\Delta u|^{2} d x \geq \alpha(2 \sigma N) \int_{\mathbb{R}^{N}}|u|^{2} d x .
$$

Now, from Gagliardo-Nirenberg inequality, we infer that for some $C>0$,

$$
1=\left(\int_{\mathbb{R}^{N}}|u|^{2 \sigma+2} d x\right)^{\frac{8}{4(2 \sigma+2)-2 \sigma N}} \leq C\left(\int_{\mathbb{R}^{N}}|\Delta u|^{2} d x\right)^{\frac{2 N}{4(2 \sigma+2)-2 \sigma N}} \int_{\mathbb{R}^{N}}|u|^{2} d x,
$$

which implies

$$
\gamma(2 N-(2 \sigma+2)(N-4))\left(\int_{\mathbb{R}^{N}}|\Delta u|^{2} d x\right)^{1+\frac{2 \sigma N}{4(2 \sigma+2)-2 \sigma N}} \geq \alpha(2 \sigma N) C .
$$

This shows that $\Delta u$ blows up in $L^{2}\left(\mathbb{R}^{3}\right)$ when $\gamma \rightarrow 0$.

\section{Sign-CHANGING RADIAL MinimizER}

In this section, we show that a radial least energy solution of (3) with $\sigma=1$ is sign-changing when $-2 \sqrt{\alpha}<\beta<2 \sqrt{\alpha}$. We assume $N=3$ but the arguments apply in dimension $N=2$ also.

We will require the decay of the radial derivatives. Arguing as in de Figueiredo et al [12, Theorem 2.2], one easily gets the following lemma.

Lemma 4. Let $u \in H_{\text {rad }}^{m}\left(\mathbb{R}^{3}\right)$ and let $\left.v:\right] 0, \infty[\rightarrow \mathbb{R}$ be the function defined by $v(r):=u(x)$ with $r=|x|$. Then, $v \in H^{m}(] 0, \infty\left[, r^{2}\right)$. Moreover, for a.e. $\left.|x| \in\right] 0, \infty[$ we have

$$
\left|D^{j} u(x)\right| \geq\left|v^{(j)}(|x|)\right|, \quad \forall j=0,1, \ldots, m .
$$

In order to prove the Theorem 4 we adapt some arguments of Bonheure et al $[2$, Theorem 6].

Proof of Theorem 4. We suppose $N=3$, the case $N=2$ is similar.

Step 1. Classical regularity. We start by observing that by elliptic regularity, we have $u \in H^{6}\left(\mathbb{R}^{3}\right)$ which implies $u \in C^{4,1 / 2}\left(\mathbb{R}^{3}\right)$ and the solution can be understood in the classical sense. Indeed, we know that the solution is $H^{2}$, so that from the equation

$$
-\Delta(-\Delta u)=|u|^{2} u-\alpha u+\beta \Delta u
$$

we infer that $-\Delta(-\Delta u) \in L^{2}\left(\mathbb{R}^{3}\right)$. This implies that $-\Delta u,-\Delta(-\Delta u) \in L^{2}\left(\mathbb{R}^{3}\right)$ and henceforth $-\Delta u \in H^{2}\left(\mathbb{R}^{3}\right)$. Since $u \in H^{2}\left(\mathbb{R}^{3}\right)$, we conclude that $u \in H^{4}\left(\mathbb{R}^{3}\right)$. Looking again at the equation, we can now use the fact that the right-hand side is an $H^{2}$-function. Then $-\Delta u,-\Delta(-\Delta u) \in H^{2}\left(\mathbb{R}^{3}\right)$ and therefore $-\Delta u \in H^{4}\left(\mathbb{R}^{3}\right)$. At last, combining the fact that $u \in H^{4}\left(\mathbb{R}^{3}\right)$ and $-\Delta u \in H^{4}\left(\mathbb{R}^{3}\right)$, we deduce that $u \in H^{6}\left(\mathbb{R}^{3}\right)$. Here above, the required elliptic regularity theory can be found in [18, Chapter 1] and since we are in the whole space, this is just a consequence of simple Fourier analysis.

Step 2. Equation in radial coordinates and decay at infinity. Writing now the equation (3) in radial coordinates (the expression is especially simple in dimension $N=3)$, we compute that $v$, defined by $v(r):=u(x)$ for $r=|x|$, solves

$$
\left.v^{i v}+\frac{4}{r} v^{\prime \prime \prime}-\beta v^{\prime \prime}-\frac{2 \beta}{r} v^{\prime}+\alpha v=|v|^{2} v, \quad r \in\right] 0, \infty[.
$$


The $H^{5}\left(\mathbb{R}^{3}\right)$ regularity yields

$$
\lim _{|x| \rightarrow \infty}\left(u(x), \partial_{x_{i}} u(x), \partial_{x_{i} x_{j}}^{2} u(x), \partial_{x_{i} x_{j} x_{k}}^{3} u(x)\right)=(0,0,0,0)
$$

whatever $i, j, k \in\{1,2,3\}$. Then Lemma 4 implies that $v$ satisfies

$$
\lim _{r \rightarrow \infty}\left(v(r), v^{\prime}(r), v^{\prime \prime}(r), v^{\prime \prime \prime}(r)\right)=(0,0,0,0)
$$

Step 3. Asymptotic analysis of the solution of the ordinary differential equation (16).

Claim 1: Given $R>0$ we can find $\bar{r} \geq R$ such that $v(\bar{r})>0$.

Let $R>0$ be fixed. Consider the following Cauchy problem

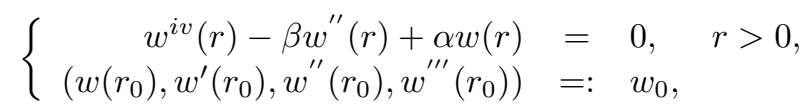

where $r_{0}>0$ and $w_{0} \in \mathbb{R}^{4}$. By using condition $(A 1)$ we have that all the roots of the characteristic equation associated to $(C 1)$ are complex, let us say $\pm a \pm i b$. We set $\Delta:=2 \pi / b$. Then there exists $c>0$ such that any solution of $(C 1)$ satisfies

$$
\sup _{\left[r_{0}, r_{0}+\Delta\right]} w, \sup _{\left[r_{0}, r_{0}+\Delta\right]}(-w) \geq c\left|w_{0}\right| .
$$

Moreover, there exists $M>0$ such that any solution of $(C 1)$ verifies

$$
\|w\|_{C^{3}\left(\left[r_{0}, r_{0}+\Delta\right]\right)} \leq M\left|w_{0}\right| .
$$

Again, we can also find $N>0$ such that the solutions of

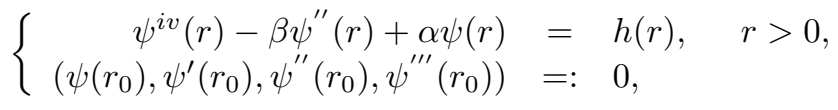

satisfy

$$
\|\psi\|_{C^{3}\left(\left[r_{0}, r_{0}+\Delta\right]\right)} \leq N\|h\|_{L^{\infty}\left(r_{0}, r_{0}+\Delta\right)} .
$$

Let us set $\delta>0$ so that $c-\frac{M N \delta}{1-N \delta}>0$. Denote by $v(r)=v\left(r ; r_{0}, v_{0}\right)$ the solution of (16) with initial conditions

$$
\left(v\left(r_{0}\right), v^{\prime}\left(r_{0}\right), v^{\prime \prime}\left(r_{0}\right), v^{\prime \prime \prime}\left(r_{0}\right)\right)=: v_{0}, \quad \text { where } r_{0}>0 .
$$

Now, let us fix $r_{0} \geq R$ large enough so that $\left|v_{0}\right|$ is small enough to have

$$
\sup _{r \in\left[r_{0}, r_{0}+\Delta\right]}|v(r)|^{2}, \sup _{r \in\left[r_{0}, r_{0}+\Delta\right]} \frac{4}{r} \text { and } \sup _{r \in\left[r_{0}, r_{0}+\Delta\right]} \frac{2 \beta}{r}<\delta \text {. }
$$

We write

$$
v=\psi+w,
$$

where $\psi$ solves

$$
\left\{\begin{aligned}
\psi^{i v}-\beta \psi^{\prime \prime}+\alpha \psi & =|v|^{2} v+\frac{2 \beta}{r} v^{\prime}-\frac{4}{r} v^{\prime \prime \prime}, r>0, \\
\left(\psi\left(r_{0}\right), \psi^{\prime}\left(r_{0}\right), \psi^{\prime \prime}\left(r_{0}\right), \psi^{\prime \prime \prime}\left(r_{0}\right)\right) & =0,
\end{aligned}\right.
$$

and $w$ is a solution of

$$
\left\{\begin{aligned}
w^{i v}(r)-\beta w^{\prime \prime}(r)+\alpha w(r) & =0, \quad r>0, \\
\left(w\left(r_{0}\right), w^{\prime}\left(r_{0}\right), w^{\prime \prime}\left(r_{0}\right), w^{\prime \prime \prime}\left(r_{0}\right)\right) & =v_{0} .
\end{aligned}\right.
$$

Now, let us choose $\bar{r} \in\left[r_{0}, r_{0}+\Delta\right]$ such that

$$
w(\bar{r}) \geq c\left|v_{0}\right| .
$$

Thus,

$$
\|\psi\|_{C^{3}\left(\left[r_{0}, r_{0}+\Delta\right]\right)} \leq N \delta\|v\|_{C^{3}\left(\left[r_{0}, r_{0}+\Delta\right]\right)},
$$


which implies that

$$
\|\psi\|_{C^{3}\left(\left[r_{0}, r_{0}+\Delta\right]\right)} \leq \frac{N \delta}{1-N \delta}\|w\|_{C^{3}\left(\left[r_{0}, r_{0}+\Delta\right]\right)} \leq \frac{M N \delta}{1-N \delta}\left|v_{0}\right| .
$$

Then we obtain

$$
v(\bar{r}) \geq c\left|v_{0}\right|-\|\psi\|_{L^{\infty}} \geq\left(c-\frac{M N \delta}{1-N \delta}\right)\left|v_{0}\right|>0 .
$$

Claim 2: Given $R>0$ we can find $\underline{r} \geq R$ such that $v(\underline{r})<0$.

The proof of this claim is similar to that of Claim 1.

Conclusion. We have proved in the last step that $u$ changes sign. In fact, we have even proved that $u$ oscillate as $|x| \rightarrow+\infty$.

\section{Comments}

This note provides some simple results for the model equation (3) with a Kerr nonlinearity and aims to partially complete the discussion on waveguide solutions in [11, Section 4.1]. The methods we used are standard. On the other hand, since radial solutions present oscillations for $-2 \sqrt{\alpha}<\beta<2 \sqrt{\alpha}$, we expect that one needs new arguments to answer the question whether the least energy solutions are radial or not in this case. Also uniqueness is a challenging question if we are not in the asymptotic regime $\beta \rightarrow \infty$ (or equivalently $\gamma \rightarrow 0$ ).

We also mention that the important question about the decay at infinity of the least energy solutions will be addressed in a future work. We are only aware of [10] for a result in that direction. The analysis therein relies on the computation of the fundamental solution of the fourth-order operator in (3) with $\beta=0$.

The analysis of the decay should also allow to extend the statement of Theorem 3 to the case $2<2 \sigma+2<\frac{2 N}{N-2}$ and $N \geq 3$. Indeed, the arguments we used are just fine for the Kerr nonlinearity whereas some technical adjustments are needed for a general subcritical power. In fact, one checks easily that our arguments apply in dimension $N \leq 4$ if we assume $2 \leq 2 \sigma+1 \leq \frac{N}{N-2}$. The lower inequality on $\sigma$ implies the required $C^{1,1}$ regularity of the function $s \mapsto|s|^{2 \sigma} s$ whereas the upper inequality is used to start the bootstrap with a $L^{2}$-bound on $|u|^{2 \sigma} u$ (here $u$ is a solution).

The same remark holds for Theorem 4 which should be true with less restrictive assumptions. In dimension $N \leq 8$, one can deal with $2 \leq 2 \sigma+1 \leq \frac{N}{N-4}$. The other cases will require more care and will be treated in a forthcoming work.

\section{ACKNOWLEDGMENTS}

D. Bonheure is supported by INRIA - Team MEPHISTO, MIS F.4508.14 (FNRS), PDR T.1110.14F (FNRS) \& ARC AUWB-2012-12/17-ULB1- IAPAS.

\section{REFERENCES}

[1] M. Ben-Artzi, H. Koch, and J. C. Saut, Dispersion estimates for fourth order Schrödinger equations, C. R. Acad. Sci. Paris Sér. I Math., 330, 87-92, 2000.

$[2]$ D. Bonheure, P. Habets and L. Sanchez, Heteroclinics for fourth order symmetric bistable equations, Atti. del Seminario Matematico e Fisico dell'Universita di Modena, 52, 213-227, 2004.

[3] D. Bonheure, E. Moreira dos Santos and M. Ramos, Ground state and non-ground solutions of some strongly coupled elliptic systems, Trans. Amer. Math. Soc. 364, 447-491, 2012.

[4] D. Bonheure, E. Moreira dos Santos and H. Tavares, Hamiltonian elliptic systems: a guide to variational frameworks, Portugal. Math. (N.S.) 71, Fasc. 3-4, 301-395, 2014.

[5] O. Bouchel, Remarks on NLS with higher order anisotropic dispersion, Adv. Differential Equations 13, 1-2, 169-198, 2008. 
[6] T. Boulenger and E. Lenzmann, Blowup for Biharmonic NLS, arXiv:1503.01741v1.

[7] J. Busca and B. Sirakov, Results for Semilinear Elliptic Systems in the Whole Space, J. Diff. Equations, 163, 41-56, 2000.

[8] S. M. Chang, S. Gustafson, K. Nakanishi and T. P. Tsai, Spectra of linearized operators for NLS solitary waves, SIAM J. Math. Anal., 39, 1070-1111, 2008.

[9] C. V. Coffman, Uniqueness of the ground state solution for $\Delta u-u+u^{3}=0$ and a variational characterization of other solutions, Arch. Rational Mech. Analysis, 46, 81-95, 1972.

[10] Y.B. Deng and Y. Li, Exponential decay of the solutions for nonlinear biharmonic equations, Communications in Contemporary Mathematics, 9, 753-768, 2007.

[11] G. Fibich, B. Ilan and G. Papanicolaou, Self-focusing with fourth order dispersion, SIAM J. Appl. Math., 62, 1437-1462, 2002.

[12] D. G. de Figueiredo, E. Moreira dos Santos and O. H. Miyagaki, Sobolev spaces of symmetric functions and applications, J. Funct. Anal., 261, 3735-3770, 2011.

[13] D. G. de Figueiredo and J. Yang, Decay, symmetry and existence of solutions of semilinear elliptic systems, Nonlinear Anal., 33, 211-234, 1998.

[14] D. Gilbarg and N. S. Trudinger, Elliptic Partial Differential Equations of Second Order, Springer, 1998.

[15] Y. Kabeya and K. Tanaka, Uniqueness of positive radial solutions of semilinear elliptic equations in $\mathbb{R}^{N}$ and Séré's non-degeneracy condition, Comm. Partial Differential Equations, 24, 563-598, 1999

[16] I. Karpman and A. G. Shagalov, Stability of solitons described by nonlinear Schrödinger-type equations with higher-order dispersion, Phys. D, 144, 194-210, 2000.

[17] O. Kavian, Introduction à la théorie des points critiques, Springer, 1993.

[18] N. V. Krylov, Lectures on Elliptic and Parabolic Equations in Sobolev Spaces, Graduate Studies in Mathematics, 96, AMS, 2008.

[19] M. K. Kwong, Uniqueness of positive solutions of $-\Delta u+u=u^{p}$ in $\mathbb{R}^{N}$, Arch. Rat. Mech. Anal., 105, 243-266, 1989.

[20] P. L. Lions, Symétrie et compacité dans les espaces de Sobolev, J. Funct. Anal., 49, 315-334, 1982.

[21] P. L. Lions, The concentration-compactness principle in the calculus of variations. The locally compact case, part 1, Ann. Inst. Henri Poincaré, Analyse non-linéaire, 1, 109-145, 1984.

[22] W. A. Strauss, Existence of solitary waves in higher dimensions, Comm. Math. Phys., 57, 149-162, 1977.

[23] W. C. Troy, Properties in Systems of Semilinear Elliptic Equations, J. Differential Equations, 42, 400-413, 1981

[24] R. C. A. M. van der Vorst, Best constant for the embedding of the space $H^{2} \cap H_{0}^{1}(\Omega)$ into $L^{2 N /(N-4)}(\Omega)$, Differential Integral Equations, 6, 259-276, 1993.

[25] M. I. Weinstein, Nonlinear Schrödinger equations and sharp interpolation estimates, Comm. Math. Phys., 87, 567-576, 1983.

DÉPARTEMENT DE MAThÉMATIQUe

UNIVERsité LiBRE DE BRUXELles

CP 214, Boulevard du Triomphe,

B-1050 Bruxelles, Belgium

E-mail address: denis.bonheure@ulb.ac.be, robson.nascimento@ulb.ac.be 